\title{
Fundamentals of Plasma Physics
}


Springer Science+Business Media, LLC 
J.A. Bittencourt

\section{Fundamentals of Plasma Physics}

Third Edition 
J. A. Bittencourt

National Institute for Space Research (INPE)

Av. dos Astronautas, 1758 - Caixa Postal 515

12245-970 São José dos Campos, SP

Brazil

jabittencourt@hotmail.com

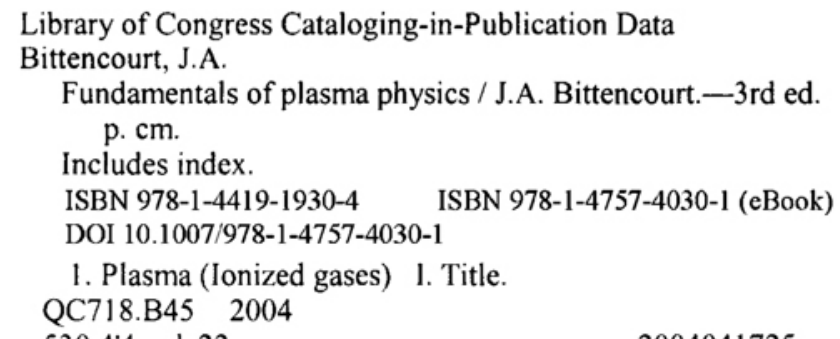

$530.4^{\prime} 4-\mathrm{dc} 22$

2004041725

Printed on acid-free paper.

(c) 2004 Springer Science+Business Media New York

Originally published by Springer-Verlag New York, Inc. in 2004

All rights reserved. This work may not be translated or copied in whole or in part without the written permission or the pubısner Springer Science+Business Media, LLC.

except for brief excerpts in connection with reviews or scholarly analysis. Use in connection with any form of information storage and retrieval, electronic adaptation, computer software, or by similar or dissimilar methodology now known or hereafter developed is forbidden.

The use in this publication of trade names, trademarks, service marks, and similar terms, even if they are not identified as such, is not to be taken as an expression of opinion as to whether or not they are subject to proprietary rights.

98766543321

springeronline.com 


\section{Preface}

This text is intended as a general introduction to plasma physics and was designed with the main purpose of presenting a comprehensive, logical, and unified treatment of the fundamentals of plasma physics based on statistical kinetic theory. It should be useful primarily for advanced undergraduate and first-year graduate students meeting the subject of plasma physics for the first time and presupposes only a basic elementary knowledge of vector analysis, differential equations, and complex variables, as well as courses on classical mechanics and electromagnetic theory beyond sophomore level. Some effort has been made to make the book self-contained by including in the text developments of fluid mechanics and kinetic theory that are needed.

Throughout the text the emphasis is on clarity, rather than formality. The various derivations are explained in detail and, wherever possible, the physical interpretations are emphasized. The equations are presented in such a way that they connect together, without requiring the reader to do extensive algebra to bridge the gap. The features of clarity and completeness make the book suitable for self-learning and for self-paced courses.

The structure of this book is as follows. Chapter 1 consists of a basic introduction to plasma physics, at a descriptive level, intended to give the reader an overall view of the subject. The motion of charged particles under the influence of specified electric and magnetic fields is treated in detail in Chapters 2, 3, and 4. In the next five chapters the fundamental equations necessary for an elementary description of plasma 
phenomena are developed. Chapter 5 introduces the concepts of phase space and distribution function, and derives the basic differential kinetic equation that governs the evolution of the distribution function in phase space. The definitions of the macroscopic variables in terms of the phase space distribution function are presented in Chapter 6 and their physical interpretations are discussed. The Maxwell-Boltzmann equilibrium distribution function is introduced in Chapter 7, as the equilibrium solution of the Boltzmann equation, and its kinetic properties are analyzed in some detail. In Chapter 8 the macroscopic transport equations for a plasma considered as a mixture of various interpenetrating fluids are derived, whereas the macroscopic transport equations for the whole plasma as a single conducting fluid are developed in Chapter 9 .

The remainder of the book is devoted to applications of these basic equations in the description of a variety of important phenomena in plasmas. The problems of electrical conductivity and diffusion in plasmas are analyzed in Chapter 10, and other basic plasma phenomena, such as electron plasma oscillations and Debye shielding, are treated in Chapter 11. Simple applications of the magnetohydrodynamic equations, such as in plasma confinement by magnetic fields and the pinch effect, are presented in Chapters 12 and 13. The subject of wave phenomena in plasmas is organized in the next six chapters. A review of the basic concepts related to electromagnetic wave propagation in free space is given in Chapter 14 . The propagation of very low frequency waves in a highly conducting fluid is analyzed in Chapter 15, under the title of magnetohydrodynamic waves. The various modes of wave propagation in cold and warm plasmas are considered in Chapters 16 and 17, respectively. In Chapters 18 and 19 the various properties of wave propagation in hot nonmagnetized plasmas and in hot magnetized plasmas, respectively, are analyzed. Collisional phenomena in plasmas are treated in Chapter 20, and the derivations of the Boltzmann collision integral and of the Fokker-Planck collision term are presented in Chapter 21. Finally, in Chapter 22 some applications of the Boltzmann equation to the analysis of transport phenomena in plasmas are presented.

Problems are provided at the end of each chapter, which illustrate additional applications of the theory and supplement the textual material. Most of the problems are designed in such a way as to provide a guideline for the student, including intermediate steps and answers in their statements.

The numbering of the equations, within each chapter, starts over again at each section. When reference is made to an equation using three 
numbers, the first number indicates the chapter and the last two numbers indicate the section and the equation, respectively. Within the same chapter the first number is omitted. Vector quantities are represented by boldface type letters (such as $\mathbf{r}$ ) and unit vectors by a circumflex above the corresponding letter (such as $\widehat{\mathbf{r}}$ ). Dyadic and triadic quantities are represented by calligraphic type letters (such as $\mathcal{Q}$ ).

The system of units used in this text is the rationalized MKSA. This system is based on four primary quantities: length, mass, time, and current. Its name derives from the units meter $(\mathrm{m})$, kilogram $(\mathrm{kg})$, second (s), and ampere (A).

The book contains more material than what can normally be covered in one semester. This permits some freedom in the selection of topics depending on the level and desired emphasis of the course, and on the interests of the students. The whole text can also be adequately covered within two semesters.

In this, as in any introductory book, the topics included clearly do not cover all areas of plasma physics. No attempt was made to present the experimental aspects of the subject. Moreover, there are some important theoretical topics that are covered only very briefly and some that have been left for more advanced courses on plasma physics, such as plasma instabilities, plasma radiation, nonlinear plasma theory, and plasma turbulence.

I am grateful to the many people who contributed to this book, both directly and indirectly, and especially to the many students to whom I had the opportunity to test my ideas in the various courses I taught over the last twenty-five years. The amount of digitalized information in a book such as this is truly enormous, and some errors may be bound to occur. Further feedback from readers will be appreciated. I wish to thank the many professors, students, and researchers who have used the first two editions of this book, all over the world, and contributed to its improvement.

\section{J. A. Bittencourt}




\section{CONTEnTS}

PREFACE

\section{INTRODUCTION}

1. General Properties of Plasmas 1

1.1 Definition of a Plasma 1

1.2 Plasma as the Fourth State of Matter 1

1.3 Plasma Production 2

1.4 Particle Interactions and Collective Effects 3

1.5 Some Basic Plasma Phenomena 4

2. Criteria for the Definition of a Plasma 6

2.1 Macroscopic Neutrality 6

$\begin{array}{lll}2.2 & \text { Debye Shielding } & 7\end{array}$

2.3 The Plasma Frequency 9

3. The Occurrence of Plasmas in Nature $\quad 11$

3.1 The Sun and its Atmosphere 13

3.2 The Solar Wind 13

3.3 The Magnetosphere and the Van Allen Radiation Belts 14

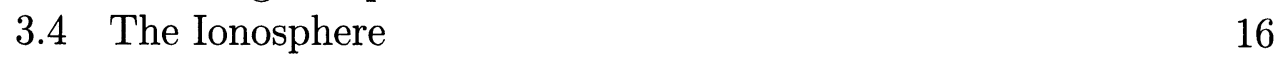

3.5 Plasmas Beyond the Solar System 17

4. Applications of Plasma Physics $\quad 17$

4.1 Controlled Thermonuclear Fusion 18

4.2 The Magnetohydrodynamic Generator 22

4.3 Plasma Propulsion 23

4.4 Other Plasma Devices 23 
5. Theoretical Description of Plasma Phenomena

5.1 General Considerations on a Self-Consistent Formulation 25

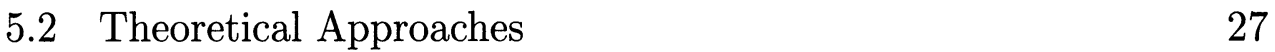

$\begin{array}{ll}\text { Problems } & 28\end{array}$

2. CHARGED PARTICLE MOTION IN CONSTANT AND UNIFORM ELECTROMAGNETIC FIELDS

1. Introduction 33

2. Energy Conservation $\quad 34$

3. Uniform Electrostatic Field 36

4. Uniform Magnetostatic Field $\quad 37$

4.1 Formal Solution of the Equation of Motion 37

4.2 Solution in Cartesian Coordinates 40

4.3 Magnetic Moment 44

4.4 Magnetization Current 46

5. Uniform Electrostatic and Magnetostatic Fields 49

5.1 Formal Solution of the Equation of Motion 49

5.2 Solution in Cartesian Coordinates 52

6. Drift Due to an External Force 54

$\begin{array}{ll}\text { Problems } & 56\end{array}$

\section{CHARGED PARTICLE MOTION IN NONUNIFORM MAGNETOSTATIC FIELDS}

1. Introduction $\quad 59$

2. Spatial Variation of the Magnetic Field 61

2.1 Divergence Terms 62

2.2 Gradient and Curvature Terms $\quad 64$

2.3 Shear Terms 65

3. Equation of Motion in the First-Order Approximation 66

4. Average Force Over One Gyration Period 68

$\begin{array}{lll}4.1 & \text { Parallel Force } & 70\end{array}$

$\begin{array}{lll}4.2 & \text { Perpendicular Force } & 72\end{array}$

4.3 Total Average Force $\quad 73$ 
6. Parallel Acceleration of the Guiding Center

6.1 Invariance of the Orbital Magnetic Moment and of the Magnetic Flux

6.2 Magnetic Mirror Effect

6.3 The Longitudinal Adiabatic Invariant

7. Curvature Drift

\section{CHARGED PARTICLE MOTION IN}

2. Slowly Time-Varying Electric Field

3. Electric Field with Arbitrary Time Variation

3.1 Solution of the Equation of Motion

3.2 Physical Interpretation

3.3 Mobility Dyad

3.4 Plasma Conductivity Dyad

3.5 Cyclotron Resonance

4. Time-Varying Magnetic Field and

4.2 Magnetic Heating of a Plasma

5. Summary of Guiding Center Drifts and

Current Densities

5.1 Guiding Center Drifts

5.2 Current Densities 


\section{ELEMENTS OF PLASMA KINETIC THEORY}

1. Introduction

2. Phase Space

2.1 Single-Particle Phase Space

2.2 Many-Particle Phase Space

2.3 Volume Elements

3. Distribution Function

4. Number Density and Average Velocity

5. The Boltzmann Equation

5.1 Collisionless Boltzmann Equation

5.2 Jacobian of the Transformation in Phase Space

5.3 Effects of Particle Interactions

6. Relaxation Model for the Collision Term

7. The Vlasov Equation

\section{AVERAGE VALUES AND MACROSCOPIC VARIABLES}

1. Average Value of a Physical Quantity

2. Average Velocity and Peculiar Velocity

3. Flux

4. Particle Current Density

5. Momentum Flow Dyad or Tensor

6. Pressure Dyad or Tensor

6.1 Concept of Pressure

6.2 Force per Unit Area

6.3 Force per Unit Volume

6.4 Scalar Pressure and Absolute Temperature

7. Heat Flow Vector

8. Heat Flow Triad

9. Total Energy Flux Triad 


\section{THE EQUILIBRIUM STATE}

1. The Equilibrium State Distribution Function

1.1 The General Principle of Detailed Balance and Binary Collisions

1.2 Summation Invariants

1.3 Maxwell-Boltzmann Distribution Function

1.4 Determination of the Constant Coefficients

1.5 Local Maxwell-Boltzmann Distribution Function

2. The Most Probable Distribution

3. Mixture of Various Particle Species

4. Properties of the Maxwell-Boltzmann

Distribution Function

4.1 Distribution of a Velocity Component 171

4.2 Distribution of Speeds 174

4.3 Mean Values Related to the Molecular Speeds 176

4.4 Distribution of Thermal Kinetic Energy 178

4.5 Random Particle Flux 178

4.6 Kinetic Pressure and Heat Flux 181

5. Equilibrium in the Presence of an External Force 181

6. Degree of Ionization in Equilibrium and the Saha Equation

\section{MACROSCOPIC TRANSPORT EQUATIONS}

1. Moments of the Boltzmann Equation

2. General Transport Equation

3. Conservation of Mass

3.2 Derivation by the Method of Fluid Dynamics 
4. Conservation of Momentum

4.1 Derivation of the Equation of Motion 200

4.2 The Collision Term 203

5. Conservation of Energy 204

5.1 Derivation of the Energy Transport Equation 204

5.2 Physical Interpretation 207

5.3 Simplifying Approximations 207

6. The Cold Plasma Model 210

7. The Warm Plasma Model 211

$\begin{array}{ll}\text { Problems } & 212\end{array}$

\section{MACROSCOPIC EQUATIONS FOR A CONDUCTING FLUID 219}

1. Macroscopic Variables for a

Plasma as a Conducting Fluid $\quad 219$

2. Continuity Equation 222

3. Equation of Motion 223

4. Energy Equation $\quad 224$

5. Electrodynamic Equations for a Conducting Fluid $\quad \mathbf{2 2 7}$

5.1 Maxwell Curl Equations 228

5.2 Conservation of Electric Charge 228

5.3 Generalized Ohm's Law 229

6. Simplified Magnetohydrodynamic Equations 234

Problems $\quad 236$

\section{PLASMA CONDUCTIVITY AND DIFFUSION 238}

1. Introduction $\quad 238$

2. The Langevin Equation $\quad 238$

3. Linearization of the Langevin Equation 240

4. DC Conductivity and Electron Mobility 242

$\begin{array}{lll}4.1 & \text { Isotropic Plasma } & 242\end{array}$

4.2 Anisotropic Magnetoplasma 243 
5. AC Conductivity and Electron Mobility

6. Conductivity with Ion Motion

7. Plasma as a Dielectric Medium

250

8. Free Electron Diffusion

251

9. Electron Diffusion in a Magnetic Field

254

10. Ambipolar Diffusion

11. Diffusion in a Fully Ionized Plasma 260

Problems

262

\section{SOME BASIC PLASMA PHENOMENA}

2. The Debye Shielding Problem

3. Debye Shielding Using the Vlasov Equation

4. Plasma Sheath

4.2 Electric Potential on the Wall

5. Plasma Probe

\section{SIMPLE APPLICATIONS OF MAGNETOHYDRODYNAMICS}

1. Fundamental Equations of Magnetohydrodynamics

1.1 Parker Modified Momentum Equation

1.2 The Double Adiabatic Equations of Chew, Goldberger, and Low (CGL)

1.3 Special Cases of the Double Adiabatic Equations

1.4 Energy Integral

2. Magnetic Viscosity and Reynolds Number 
4. Freezing of Magnetic Field Lines to the Plasma

5. Magnetic Pressure

6. Isobaric Surfaces

7. Plasma Confinement in a Magnetic Field

\section{THE PINCH EFFECT}

1. Introduction

2. The Equilibrium Pinch

3. The Bennett Pinch

4. Dynamic Model of the Pinch

335

5. Instabilities in a Pinched Plasma Column

341

6. The Sausage Instability

7. The Kink Instability

8. Convex Field Configurations

\section{ELECTROMAGNETIC WAVES IN FREE SPACE}

1. The Wave Equation

2. Solution in Plane Waves

3. Harmonic Waves

4. Polarization

5. Energy Flow

6. Wave Packets and Group Velocity 


\section{MAGNETOHYDRODYNAMIC WAVES}

1. Introduction

1.2 Magnetosonic Waves

2. MHD Equations for a Compressible

Nonviscous Conducting Fluid

2.1 Basic Equations

2.2 Development of an Equation for the Fluid Velocity

3. Propagation Perpendicular to the Magnetic Field

4. Propagation Parallel to the Magnetic Field

5. Propagation at Arbitrary Directions

5.1 Pure Alfvén Wave

5.2 Fast and Slow MHD Waves

5.3 Phase Velocities

5.4 Wave Normal Surfaces

6. Effect of Displacement Current

6.1 Basic Equations

6.2 Equation for the Fluid Velocity

392

6.3 Propagation Across the Magnetostatic Field

393

6.4 Propagation Along the Magnetostatic Field

7. Damping of MHD Waves

\section{WAVES IN COLD PLASMAS}

2. Basic Equations of Magnetoionic Theory

3. Plane Wave Solutions and Linearization

4. Wave Propagation in Isotropic Electron Plasmas 
4.3 Time-Averaged Poynting Vector 407

4.4 The Effect of Collisions 410

5. Wave Propagation in Magnetized Cold Plasmas 413

5.1 Derivation of the Dispersion Relation 414

5.2 The Appleton-Hartree Equation 418

6. Propagation Parallel to $B_{0} \quad 419$

7. Propagation Perpendicular to $B_{0}$

8. Propagation at Arbitrary Directions 430

8.1 Resonances and Reflection Points 430

8.2 Wave Normal Surfaces 432

8.3 The CMA Diagram 434

9. Some Special Wave Phenomena in Cold Plasmas 439

9.1 Atmospheric Whistlers 439

9.2 Helicons 442

9.3 Faraday Rotation 444

$\begin{array}{ll}\text { Problems } & 447\end{array}$

\section{WAVES IN WARM PLASMAS 453}

1. Introduction 453

2. Waves in a Fully Ionized Isotropic Warm Plasma 453

2.1 Derivation of the Equations for the Electron and Ion Velocities 453

2.2 Longitudinal Waves 456

2.3 Transverse Wave 458

3. Basic Equations for Waves in a Warm Magnetoplasma 460

4. Waves in a Warm Electron Gas in a Magnetic Field 462 4.1 Derivation of the Dispersion Relation 462

4.2 Wave Propagation Along the Magnetic Field 463

4.3 Wave Propagation Normal to the Magnetic Field 466

4.4 Wave Propagation at Arbitrary Directions 469

5. Waves in a Fully Ionized Warm Magnetoplasma $4 \mathbf{4 7 0}$

5.1 Derivation of the Dispersion Relation 471

5.2 Wave Propagation Along the Magnetic Field 473

5.3 Wave Propagation Normal to the Magnetic Field 477 
6. Summary

\section{WAVES IN HOT ISOTROPIC PLASMAS}

1. Introduction

2. Basic Equations

3. General Results for a Plane Wave in a Hot Isotropic Plasma

3.1 Perturbation Charge Density and Current Density 485

3.2 Solution of the Linearized Vlasov Equation 486

3.3 Expression for the Current Density 488

3.4 Separation into the Various Modes 489

4. Electrostatic Longitudinal Wave in a Hot Isotropic Plasma

4.1 Development of the Dispersion Relation 491

4.2 Limiting Case of a Cold Plasma 492

4.3 High Phase Velocity Limit 493

4.4 Dispersion Relation for

Maxwellian Distribution Function 494

4.5 Landau Damping $\quad 500$

5. Transverse Wave in a Hot Isotropic Plasma 503

5.1 Development of the Dispersion Relation 503

5.2 Cold Plasma Result 504

5.3 Dispersion Relation for

Maxwellian Distribution Function 504

5.4 Landau Damping of the Transverse Wave 505

6. The Two-Stream Instability 506

7. Summary $\quad 508$

$\begin{array}{ll}7.1 & \text { Longitudinal Mode } \\ 708\end{array}$

$\begin{array}{lll}7.2 & \text { Transverse Mode } & 509\end{array}$ 


\section{WAVES IN HOT MAGNETIZED PLASMAS}

1. Introduction

2. Wave Propagation Along the

Magnetostatic Field in a Hot Plasma

2.1 Linearized Vlasov Equation

2.2 Solution of the Linearized Vlasov Equation

2.3 Perturbation Current Density

2.4 Separation into the Various Modes

2.5 Longitudinal Plasma Wave

2.6 Transverse Electromagnetic Waves

2.7 Temporal Damping of the Transverse Electromagnetic Waves

2.8 Cyclotron Damping of the RCP Transverse Wave

2.9 Instabilities in the RCP Transverse Wave

3. Wave Propagation Across the

Magnetostatic Field in a Hot Plasma

3.1 Solution of the Linearized Vlasov Equation

3.2 Current Density and the Conductivity Tensor

538

3.3 Evaluation of the Integrals

540

3.4 Separation into the Various Modes

3.5 Dispersion Relations

545

3.6 The Quasistatic Mode

546

3.7 The TEM Mode

550

4. Summary

4.2 Propagation Across $\mathbf{B}_{0}$ in Hot Magnetoplasmas

1. Introduction

2. Binary Collisions

3. Dynamics of Binary Collisions 
4.2 Coulomb Interaction Potential 570

5. Cross Sections

5.1 Differential Scattering Cross Section 574

5.2 Total Scattering Cross Section 576

5.3 Momentum Transfer Cross Section 577

6. Cross Sections for the Hard Sphere Model 578

6.1 Differential Scattering Cross Section 578

6.2 Total Scattering Cross Section 579

6.3 Momentum Transfer Cross Section 579

7. Cross Sections for the Coulomb Potential $\mathbf{5 8 0}$

7.1 Differential Scattering Cross Section 580

7.2 Total Scattering Cross Section 581

7.3 Momentum Transfer Cross Section 581

8. Screening of the Coulomb Potential 582

$\begin{array}{ll}\text { Problems } & 586\end{array}$

\section{THE BOLTZMANN AND THE FOKKER-PLANCK EQUATIONS 589}

1. Introduction

2. The Boltzmann Equation $\quad \mathbf{5 9 0}$

2.1 Derivation of the Boltzmann Collision Integral 590

2.2 Jacobian of the Transformation 594

2.3 Assumptions in the Derivation of the Boltzmann Collision Integral $\quad 596$

2.4 Rate of Change of a Physical Quantity as a Result of Collisions $\quad 597$

3. The Boltzmann's H Function $\quad 598$

3.1 Boltzmann's H Theorem 599

3.2 Analysis of Boltzmann's H Theorem 601

3.3 Maximum Entropy or Minimum H Approach
for Deriving the Equilibrium Distribution Function

3.4 Mixture of Various Particle Species 606

4. Boltzmann Collision Term 
4.1 Spherical Harmonic Expansion of the Distribution Function

4.2 Approximate Expression for the Boltzmann Collision Term

4.3 Rate of Change of Momentum Due to Collisions

5.1 Derivation of the Fokker-Planck Collision Term

5.2 The Fokker-Planck Coefficients for Coulomb Interactions

5.3 Application to Electron-Ion Collisions

\section{Problems}

\section{TRANSPORT PROCESSES IN PLASMAS}

1. Introduction

2. Electric Conductivity in a Nonmagnetized Plasma

2.2 Electric Current Density and Conductivity

2.3 Conductivity for Maxwellian Distribution Function

3. Electric Conductivity in a Magnetized Plasma

3.2 Electric Current Density and Conductivity

4.1 Perturbation Distribution Function

4.2 Particle Flux

4.3 Free Diffusion Coefficient

5. Diffusion in a Magnetic Field

5.1 Solution of Boltzmann Equation 643

5.2 Particle Flux and Diffusion Coefficients 645

6. Heat Flow

6.1 General Expression for the Heat Flow Vector 647

6.2 Thermal Conductivity for a Constant Kinetic Pressure 648

6.3 Thermal Conductivity for the Adiabatic Case 649 


\section{APPENDIX A}

Useful Vector Relations

655

APPENDIX B

Useful Relations in Cartesian and in Curvilinear Coordinates

APPENDIX C

Physical Constants (MKSA)

APPENDIX D

Conversion Factors for Physical Units

APPENDIX E

Some Important Plasma Parameters

APPENDIX F

Approximate Magnitudes in Some Typical Plasmas

INDEX 